\title{
Evaluation of Family Dysfunction in Patients with Schizophrenia and Bipolar I Disorder
}

\author{
Peyman Hashemian1, Mohammad Edris Sedaghati2* \\ ${ }^{1}$ Psychiatry and Behavioral Sciences Research Center, Ibn-e-Sina Hospital, Faculty of Medicine, \\ Mashhad University of Medical Sciences, Mashhad, Iran \\ ${ }^{2}$ Unit of Family Medicine, Faculty of Medicine, North Khorasan University of Medical Sciences, Bojnurd, Iran \\ Email: "edriss_6385@yahoo.com
}

Received 23 November 2015; accepted 13 February 2016; published 16 February 2016

Copyright (C) 2016 by authors and Scientific Research Publishing Inc.

This work is licensed under the Creative Commons Attribution International License (CC BY). http://creativecommons.org/licenses/by/4.0/

(c) (i) Open Access

\begin{abstract}
Introduction: Schizophrenia and bipolar I disorder are very common disorders in hospitalized patients. Considering that family problems are one of the factors in the appearance and persistence of schizophrenia and bipolar I disorder, in this study, we decided to investigate and compare the family functioning between these two groups of patients. Methods: The sample consisted of 50 patients with schizophrenia and 50 patients with bipolar I disorder. The third group was the control group which consisted of $\mathbf{5 0}$ normal different professions such as teachers, workers, housekeepers and others. Then the Family Functioning Scale (FAD-I) was used and the final results of the three groups were compared by SPSS V21 software. Results: In the schizophrenia group, 88.9\% percent of patients had family functioning scores less than 109.81 which showed that they had severe family dysfunction but in the bipolar I disorder group, $11.1 \%$ and all participants in the control group, had scores higher than 109.81.Conclusion: Family dysfunction was seen in schizophrenic patients rather than in the other two groups.
\end{abstract}

\section{Keywords}

Family Functioning, Schizophrenia, Bipolar I Disorder

\section{Introduction}

Family problems are one of the factors in the appearance and persistence of schizophrenia and bipolar I disorder. Also, because family dysfunction is one of the factors in schizophrenia relapse, family therapy is recommended

${ }^{*}$ Corresponding author.

How to cite this paper: Hashemian, P. and Sedaghati, M.E. (2016) Evaluation of Family Dysfunction in Patients with Schizophrenia and Bipolar I Disorder. Journal of Biosciences and Medicines, 4, 1-5. 
in reducing relapses.

No well-controlled evidence indicates that a specific family pattern plays a causative role in the development of schizophrenia [1].

There are many kinds of family function for example the double-bind concept formulated by Gregory Bateson and Donald Jackson to describe a hypothetical family or Schism and Skewed families or Pseudomutual and Pseudohostile families are some families who suppress emotional expression. Person with schizophrenia may have parents who may behave with overt criticism, hostility, and over involvement toward them. Families with high levels of expressed emotion may have high relapse rate for schizophrenia [2].

A study which was conducted in 2014 by Koutra et al. showed that a number of social and clinical factors contributed to family environment of patients with psychosis. Identifying the determinants of family functioning in psychosis is instrumental in developing understandings regarding the factors which may contribute to the rehabilitation or relapse of the patient and the support required to strengthen positive family interactions [3].

Another study by koutra et al. in 2014 suggested that unbalanced levels of cohesion and flexibility, high criticism and burden appeared to be the outcome of psychosis and not risk factors triggering the onset of the illness. Furthermore, emotional over-involvement both in terms of positive (i.e. concern) and negative behaviors (i.e. overprotection) was prevalent in Greek families. Psychoeducational interventions from the early stages of the illness should be considered to promote caregivers' awareness regarding patients' illness, which in turn, may ameliorate dysfunctional family interactions [4].

In a study performed by Sullivan and his colleagues in 2012 under the name "Family functioning and the course of adolescent bipolar disorder" revealed that decrease in parent-reported conflicts decreased adolescents' manic symptoms over a 2-year study. Findings suggest that family cohesion, adaptability, and conflict may be useful predictors of the course of adolescent mood symptoms [5].

In a study that has been done in 2013 by Vynstak et al. degree of concordance between patient- and family-reported family functioning was significantly weaker in bipolar disorder. Subsequent analysis revealed that this discordance was driven by the reports of child and young adolescent family members of the patients with bipolar disorder. Results highlight the importance of collateral reports in the assessment of family functioning, especially among families of patients with bipolar disorder, in research and treatment [6].

In a study done by Hassan A. Hussein and his associates in 2012, significant association between patients' clinical characteristics and caregivers' socio-demographic characteristics with family functioning were seen. The researchers recommend conducting longitudinal studies on family caregivers, using psychiatric and family assessment/intervention for those caregivers with family dysfunction [7].

A study by Deboard and his colleagues in 2010 was performed on adolescent patients with bipolar I disorder, Data suggest that supportive, responsive parenting can buffer the effects of inter-parental conflict on children by reducing self-blaming attributions for parental discord [8].

\section{Method}

In this study we decided to investigate family dysfunction between these two groups of patients with schizophrenia and bipolar I disorder.

This study was a case-control and the sample consisted of patients who were hospitalized for schizophrenia and bipolar I disorder at Ibne-Sina Hospital in Mashhad in 2014 with age over 20 years old. They were confirmed by psychiatrists and they have an IQ above 70 which were determined by Wechsler intelligence scale. They had no personality or any other psychiatric disorders.

Data were collected through interviews with family members of patients with schizophrenia and bipolar I disorder by using the Family Questionnaire (FAD-I). Control group was randomly selected in a way that they had no significant psychiatric disorder in The Symptom Ckecklist $90^{\circ}$ (SCL-90) and then interviews were conducted by the Family assessment Questionnaire (FAD-I). Families with scores less than 109.81 were considered to have severe family dysfunction.

Symptom Check List-90 (SCL-90) questionnaire is one of the psychiatric diagnostic tools [9].

In analyzing the data, the appropriate tests were used. Normality of distribution was studied by using onesample Kolmogorov-Smirnov test. In cases of non-normal distribution Kruskal-Walis test was used. SPSS version 21 software was used in this study and the significance level of test was considered less than $5 \%$. 
Ethical considerations: patient information was kept confidential.

\section{Results}

There was no significance difference on the basis of sex between three groups (P-value $=0.683$ ), (Table 1 ).

Mean age in control group, Schizophrenia and Bipolar I disorder groups were 34.9, 35.3, 37.3 respectively (Kruskal-Walis $=1 / 499$, P-value $=0 / 473$ ) in which there was no significance difference on the basis of age between groups.

In family function test, mean difference between the three groups was significant using Kruskal-Walis test (74.361, P-value $=0.0001)$. It was also significant between schizophrenia and control group $(\mathrm{P}$-value $=0.0001)$ and between bipolar and control group (P-value $=0.0001$ ). Comparison of means of family function test between schizophrenia and bipolar groups show significant difference using independent t-test (t-test $=4.720$, P-value $=0.0001)$.

After comparing the three groups on the basis of severe family dysfunction (FAD < 109.81), the following results achieved:

The frequency of severe family dysfunction (FAD < 109.81) in all the three groups were: 141 patients $(94 \%)$ with no impairment and 9 (6\%) had impairment. Based on the present study, we examined the levels of family dysfunction which were not statistically significant $(\mathrm{P}$-value $=0.329)$. The difference in the average age of people who have severe family dysfunction and those who do not have severe dysfunction is not statistically significant $(\mathrm{P}$-value $=0.837)$.

According to Table 2, the difference in family dysfunction between the three groups (schizophrenia, bipolar I disorder and control group) was significant (P-value $=0.002$ ).

According to Table 3, comparing family dysfunction in schizophrenia and bipolar I disorder groups also shows a statistically significant difference $(\mathrm{P}$-value $=0.031)$.

There is no statistically significant difference of severe family dysfunction in schizophrenia and bipolar I disorder on the basis of sex $(\mathrm{P}$-value $=1.00)$ and age $(\mathrm{P}$-value $=0.120)$.

Table 1. Frequency of three groups on the basis of gender.

\begin{tabular}{|c|c|c|c|c|c|c|}
\hline \multirow{2}{*}{ Group } & \multicolumn{2}{|c|}{ Sum } & \multicolumn{2}{|c|}{ Female } & \multicolumn{2}{|c|}{ Male } \\
\hline & Number & $\%$ & Number & $\%$ & Number & $\%$ \\
\hline Control & 50 & 33.3 & 17 & 37.8 & 33 & 31.4 \\
\hline Schizophrenia & 50 & 33.3 & 13 & 24.5 & 37 & 38.1 \\
\hline Bipolar I disorder & 50 & 33.3 & 15 & 28.3 & 35 & 36.1 \\
\hline Sum & 150 & 100 & 45 & 100 & 105 & 100 \\
\hline Analysis & \multicolumn{6}{|c|}{ Pearson chi-square $=0 / 762, \mathrm{P}-$ Value $=0 / 683$} \\
\hline
\end{tabular}

Table 2. Distribution of family dysfunction in the studied groups.

\begin{tabular}{|c|c|c|c|c|c|c|}
\hline \multirow{2}{*}{ Family Dysfunction Group } & \multicolumn{2}{|c|}{ Yes } & \multicolumn{2}{|c|}{ No } & \multicolumn{2}{|c|}{ Total } \\
\hline & Number & Percent & Number & Percent & Number & Percent \\
\hline Control group & 0 & $0 \%$ & 50 & $35.5 \%$ & 50 & $33.3 \%$ \\
\hline Schizophrenia & 8 & $88.9 \%$ & 42 & $29.8 \%$ & 50 & $33.3 \%$ \\
\hline Bipolar I disorder & 1 & $11.1 \%$ & 49 & $34.8 \%$ & 50 & $33.3 \%$ \\
\hline Total & 9 & $100 \%$ & 141 & $100 \%$ & 150 & $100 \%$ \\
\hline The test statistic probability & \multicolumn{6}{|c|}{ Fishers exact test $=11.421, \mathrm{P}$-value $=0.002$} \\
\hline
\end{tabular}


Table 3. Distribution of family dysfunction in patients with schizophrenia and bipolar I disorder.

\begin{tabular}{ccccccc}
\hline \multirow{2}{*}{ Family Dysfunction Group } & \multicolumn{2}{c}{ Yes } & \multicolumn{2}{c}{ No } & \multicolumn{2}{c}{ Total } \\
\cline { 2 - 6 } & Number & Percent & Number & Percent & Number & Percent \\
Schizophrenia & 8 & $88.9 \%$ & 42 & $46.2 \%$ & 50 & $50 \%$ \\
Bipolar I disorder & 1 & $11.1 \%$ & 49 & $53.8 \%$ & 50 & $50 \%$ \\
Total & 9 & $100 \%$ & 91 & $100 \%$ & 100 & $100 \%$ \\
The test statistic probability & & & Fishers exact test, P-value $=0.031$ & \\
\hline
\end{tabular}

\section{Discussion}

Schizophrenia and bipolar I disorder are common in hospitalized patients and identifying their family function has an important role in their treatment plan [1].

In this study, we investigate family dysfunction in patients with schizophrenia and bipolar I disorder hospitalized in Ibne-Sina Hospital, Mashhad. The three groups were analyzed for age, sex and family dysfunction.

The results of this study shows that there was a significant difference between family function in the three groups indicating more impaired family functioning in schizophrenic patients than in patients with bipolar I disorder and control group. There was also significant difference between schizophrenic and bipolar groups.

In a study performed by Sullivan and his colleagues in 2012 in USA, family functioning data were collected from 58 families of adolescents with bipolar I disorder. In this study family dysfunction has been studied over two years by increasing periods of mania in adolescents [5].

In a study conducted in 2014 by Koutra et al in Greece with name "Family functioning in families of firstepisode psychosis patients as compared to chronic mentally ill patients and healthy controls", Family cohesion, flexibility and psychological distress were evaluated in families of 50 First Episode of Psychosis and 50 chronic patients, as well as 50 controls. Findings indicated impaired cohesion and flexibility for families of First Episode of Psychosis patients compared to controls, and lower scores for families of chronic patients compared to those of First Episode of Psychosis patients [4].

In a study done in 2013 by Weinstock L.M. et al. in USA with name "Concordance between patient and family reports of family functioning in bipolar I disorder and major depressive disorder" concordance between patientand family-reported family functioning were studied. In this study, 92 patients with bipolar I disorder and 121 patients with major depressive disorder and their families were evaluated. In the findings degree of concordance between patient- and family-reported family functioning was significantly weaker in bipolar I disorder [6].

\section{Conclusion}

In this study, family dysfunction in patients with schizophrenia and bipolar I disorder is compared with each other and with control group. According to our study, it can be said that family dysfunction in schizophrenia and bipolar I disorder is higher than in control group and also in schizophrenia is much higher than in bipolar I disorder.

\section{Recommendation}

This was a preliminary study to understand whether there is a relationship between family dysfunction in schizophrenia and bipolar I disorder or not. To know whether dysfunction as an etiologic factor is involved in these diseases or not, family therapy intervention is needed.

\section{References}

[1] Sadock, B.J., Sadock, V.A. and Ruiz, P. (2009) Kaplan \& Sodock’s Comprehensive Textbook of Psychiatry. Vol. 1, 9th Edition, Wolters Kluwer/Lippincott Williams \& Wilkins, New York, 1432-1839.

[2] Sadock, B.J. and Sadock, W.V. (2008) Kaplan \& Sadock’s Concise Textbook of Clinical Psychiatry. 3rd Edition, Wolters Kluwer/Lippincott Williams \& Wilkins, New York, 161-164. 
[3] Koutra, K., Triliva, S., Roumeliotaki, T., Lionis, C. and Vgontzas, A.N. (2014) Identifying the Socio-Demographic and Clinical Determinants of Family Functioning in Greek Patients with Psychosis. International Journal of Social Psychiatry, pii: 0020764014540151.

[4] Koutra, K., Triliva, S., Roumeliotaki, T., Stefanakis, Z., Basta, M., Lionis, C. and Vgontzas, A.N. (2014) Family Functioning in Families of First-Episode Psychosis Patients as Compared to Chronic Mentally Ill Patients and Healthy Control. Psychiatry Research, pii: S0165-1781(14)00548-4.

[5] Sullivan, A.E., Judd, C.M., Axelson, D.A. and Miklowitz, D.J. (2012) Family Functioning and the Course of Adolescent Bipolar Disorder. Behavior Therapy, 43, 837-847. http://dx.doi.org/10.1016/j.beth.2012.04.005

[6] Weinstock, L.M., Wenze, S., Munroe, M.K. and Miller, I.W. (2013) Concordance between Patient and Family Reports of Family Functioning in Bipolar I Disorder and Major Depressive Disorder. The Journal of Nervous and Mental Disease, 201, 377-383. http://dx.doi.org/10.1097/NMD.0b013e31828e1041

[7] Hussein, H.A. and Khudhiar, A.K. (2012) Family Functioning among Caregivers of Patients with Schizophrenia in Baghdad City. Karbala Journal of Medicine, 5, 1204-1209.

[8] De Board, L.R.L. and Clin, J. (2010) Interparental Conflict in Context: Exploring Relations between Parenting Processes and Children's Conflict Appraisals. Journal of Clinical Child \& Adolescent Psychology, 39, 163-175.

[9] Derogatis, L.R. and Savitz, K.L. (2000) The SCL-90-R and the Brief Symptom Inventory (BSI) in Primary Care. In:: Maruish, M.E., Ed., Handbook of Psychological Assessment in Primary Care Settings, Vol. 236, Lawrence Erlbaum Associates, Mahwah, 297-334. 